\title{
Laser Micromachining of Permalloy for Fine Metal Mask
}

\author{
Junyeon Heo', Hyungsuk Min', and Myeongkyu Lee ${ }^{1, t}$ \\ 1 Department of Materials Science and Engineering, Yonsei University, 50, Yonsei-ro, Seodaemun-gu, Seoul, 120-749, South Korea \\ \# Corresponding Author / E-mail: myeong@yonsei.ac.kr, TEL: +82-2-2123-2832, FAX: +82-2-312-5375
}

KEYWORDS: Laser micromachining, Fine metal mask, Permalloy, Organic light emitting diode

\begin{abstract}
We show that crack-free drilled structures with $25 \mu \mathrm{m}$ hole size can be fabricated in the $12 \mu$ m-thick Permalloy foil by a nanosecond ultraviolet laser with low pulse energy and high repetition rate. The number of pulses required for drilling decreased with increasing pulse energy. The obtained hole exhibited a smaller size than the laser spot, implying that only the central part of a focused Gaussian beam contributed to the drilling. Debris and burr incurring as a result of the laser micromachining could be quickly removed by etching in an $\mathrm{HCl} / \mathrm{HNO}_{3}$ solution. This made it possible to obtain a clear structure without any debris remaining on the foil. This laserdirect process may be effectively utilized for fabricating fine metal masks necessary for the production of organic light emitting diode displays and other electronic devices.
\end{abstract}

Manuscript received: March 12, 2015 / Received: April 22, 2015 / Accepted: May 7, 2015

\section{NOMENCLATURE}

$\mathrm{a}=$ directional orientation of the system

$\mathrm{h}=$ strip thickness with strip thickness and strip thickness strip thickness

\section{Introduction}

As the performance level of electronic devices such as TV and smart phones is continuously increasing by the demand of consumers, device components should also be developed to be compatible with the high-performance electronic products. Organic light emitting diode (OLED) display works without a backlight. In low ambient light conditions (such as a dark room), an OLED screen can achieve a higher contrast ratio than an liquid-crystal display (LCD), regardless of whether the LCD uses cold cathode fluorescent lamps or an LED backlight. It also has merits of fast response time and small pixel size and is compatible with transparent/flexible displays. In addition, OLED TV screens have near-perfect viewing angle. Owing to these advantages, OLED has drawn much attention for the next-generation displays. ${ }^{1-3}$ Although most display companies produce OLED screens, the OLED's market share in the overall display industry is still limited. This is attributed to the fact that the production costs are currently too high to economically develop larger screen sizes. Meanwhile, the selective deposition of red/green/blue emissive layers is an essential process in the manufacturing of OLED display. This process is carried out using a fine metal mask (FMM) with many tiny openings. The FMM is typically fabricated either by lithography combined with chemical etching or electroforming. Since the fabrication requires highcost facility and/or complicated processes, each sheet of FMM is also very expensive. Although the currently used FMMs have a pixel size of $40 \mu \mathrm{m}$ ( $240 \mathrm{ppi})$, a pixel size less than $20 \mu \mathrm{m}$ (> 300 ppi) would ultimately be required for the future high-resolution, large-area devices. FMMs have generally been made from stainless steel. For large-size devices, however, the stainless steel should be replaced by Invar ( $64 \%$ $\mathrm{Fe}-36 \% \mathrm{Ni}$ alloy), which exhibits very low thermal expansion.

Lasers have proven to be a valuable tool in many forms of materials processing ranging from patterning, ${ }^{4}$ welding, ${ }^{5}$ sintering, ${ }^{6,7}$ melting, ${ }^{8}$ printing, ${ }^{9,10}$ and etc. Laser machining is an unconventional machining process in which a laser is directed towards the work piece for machining. ${ }^{11}$ Since a laser beam has monochromatic and parallel rays, it can be focused to a very small diameter, producing an extremely high energy density at the focal point. Thus, a laser beam is suited to making accurately placed holes and can also be used to perform precision micromachining on almost all materials such as metal, ceramic, and semiconductor. An early study by Roos ${ }^{12}$ showed that a $200 \mu$ s train consisting of $0.5 \mu$ s pulses produced superior results for drilling metals than a $200 \mu$ s flat-shaped pulse. In this respect, the pulsed laser with high repetition rate can provide an efficient, alternative tool for noncontact and wear-less machining. Commercial nanosecond lasers have 
been extensively used to machine many materials. ${ }^{13-16}$ In recent years, the micromachining by means of ultrashort picosecond and femtosecond lasers has drawn increasing attention, ${ }^{17-20}$ especially for drilling deep holes and cutting substrate materials. These ultrashort laser pulses offer advantageous features such as a nearly direct solidto-vapor transition and a negligible thermal effect on the surroundings of the ablation spot. ${ }^{18}$ Consequently they have the possibility to generate high-quality structures with limited melt and burr formation. One drawback is that when vaporization is involved, a vacuum chamber is necessary to remove the produced vapor. Nanosecond lasers are still an economical source for the processing of metallic materials in the form of foil or plate, although an additional chemical etching step required to remove the deposited melt and burr may increase the overall process cost. Here, we studied laser-direct drilling of Permalloy, which is of the same type of Fe-Ni alloy as Invar but is more easily available. It also maintains fairly low thermal expansion. This research has an objective of making FMMs by directly drilling holes in Permalloy foils with a laser. Different types of nanosecond lasers have been employed to drill holes in the Permalloy foil. Compared to the conventional methods, it can reduce the fabrication cost of FMMs. Not only being effectively utilized for OLEDs, this laser-direct approach can provide a facile route to fabricate fine mask structures that would be potentially required for the next-generation devices.

\section{Experimental Procedures}

The investigated Permalloy foils $(22 \%$ Fe- $78 \% \quad \mathrm{Ni}, 12 \mu \mathrm{m}$ thickness) were supplied from E-song EMC, Inc. These foils were produced by a continuous electroforming method. Two different types of nanosecond lasers were employed for laser micromachining; one is a high-pulse energy/low-repetition rate laser at $1064 \mathrm{~nm}$ and the other, a low-pulse energy/high-repetition rate laser at $355 \mathrm{~nm}$. A pulsed Nd:YAG laser at $1064 \mathrm{~nm}$ (Model: Brilliant B, Quantel, pulse width $=5 \mathrm{~ns}$, repetition rate $=10 \mathrm{~Hz}$, maximum pulse energy $=850 \mathrm{~mJ}$, output beam diameter $=9 \mathrm{~mm}$ ) was used for fine hole processing. The output beam was focused using a plano-convex lens with focal length $(f)$ of $100 \mathrm{~mm}$. The spot size $(d)$ of the focused beam was estimated from the formula of $d=2.44 f \lambda / D$, where $\lambda$ is the laser wavelength and $D$, the size of the output beam $(9 \mathrm{~mm})$. The calculated spot size at focus was about $d=29 \mu \mathrm{m}$. The Permalloy foil was attached to a stage that moves in the $\mathrm{x}, \mathrm{y}$, and $\mathrm{z}$ directions. A slide glass was inserted between the sample and the lens to prevent potential lens damage by the desorbed material.

An ultraviolet (UV) laser at $355 \mathrm{~nm}$ was also employed (Model: Avia 355-5, Coherent Inc., power $=5 \mathrm{~W}$ at $50 \mathrm{kHz}$, pulse width $<20 \mathrm{~ns}$, repetition rate $=1 \mathrm{~Hz}-150 \mathrm{kHz}$, output beam size $=2.54 \mathrm{~mm}$ ). This diode-pumped, solid-state laser combines high repetition rate and short pulse width, enabling high-throughput processing with a minimal heat-affected zone. Laser-induced materials processing is often carried out with the sample stationed on a manual or automatic translation stage. However, it is more convenient and faster to control the beam position optically rather than mechanically. An optical scanner enables the position of a focused beam to be fast moved on the sample surface. The output laser beam was incident into a galvanometric scanner that
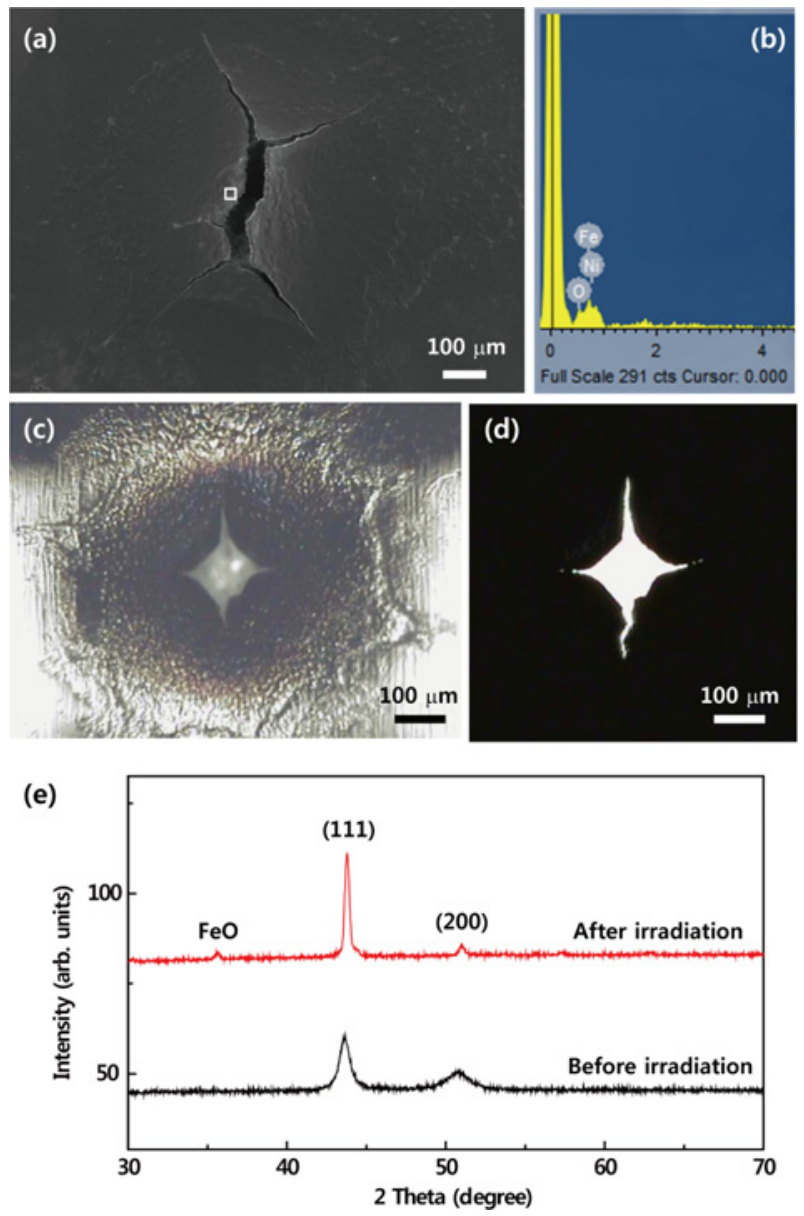

Fig. 1 (a) SEM image of a Permalloy sample irradiated by a single Nd:YAG laser pulse with $200 \mathrm{~mJ}$. (b) EDS analysis of the area marked in "(a)". (c) and (d) optical micrographs of another sample irradiated with $300 \mathrm{~mJ}$. (e) XRD patterns

steers it in the $\mathrm{x}$ and $\mathrm{y}$ directions. An F-theta lens $(f=200 \mathrm{~mm})$ was installed with the scanner to maintain a constant laser spot size on the sample surface over an area of $100 \times 100 \mathrm{~mm}^{2}$. This galvanometric scanning system, externally connected to the laser, enabled the number of pulses injected into the sample to be controlled even though the laser was operated at a high repetition rate of several $\mathrm{kHz}$. An $\mathrm{HCl} / \mathrm{HNO}_{3}$ solution was used to remove any debris and burr incurring as a result of the laser machining. The processed samples were characterized using X-ray diffraction (Rigaku diffractometer), optical microscopy (Olympus BX51), and scanning electron microscopy (JSM-7001F, JEOL Inc. $15 \mathrm{kV}$ ). All micromachining experiments were conducted in ambient atmosphere.

\section{Results and Discussion}

At first, we investigated laser drilling by a pulsed Nd:YAG laser. An output laser beam was focused onto the sample surface and the beam size at focus was estimated to be about $30 \mu \mathrm{m}$. Since this is a lowrepetition rate laser $(10 \mathrm{~Hz})$, a single pulse was used for the drilling. Fig. 1(a) shows the scanning electron microscopy (SEM) image of a sample irradiated by a pulse with energy of $200 \mathrm{~mJ}$. The sample was torned and cracked at this pulse energy. The cracking is attributed to 
laser-induced thermal shock. Thermal shock occurs when a thermal gradient causes different parts of an object to expand by different amounts. This differential expansion can be understood in terms of stress or of strain, equivalently. At some point, this stress can exceed the strength of the material, causing a crack to form. Taking the estimated beam size of $30 \mu \mathrm{m}$ into account, the energy density at focus (i.e., on the sample surface) was calculated to be $\sim 300 \mathrm{~J} / \mathrm{mm}^{2}$. It seemed that a high-energy beam focused on the surface caused abrupt heating and thermal expansion. Brittle materials such as glass are particularly sensitive to the thermal cracking. Qualitative compositional analysis was carried out by energy dispersive X-ray spectroscopy (EDS). While only Fe and Ni components were detected well outside the irradiated area, $\mathrm{O}$ component was observed in the vicinity of the cracked region, as shown in Fig. 1(b). This implies that any oxide phase formed at high temperature might have fostered the generation and propagation of cracks. Fig. 1(c) shows the reflection-mode optical micrograph of a sample irradiated with a single pulse of $300 \mathrm{~mJ}$. Although the foil was instantaneously perforated due to the increased pulse energy, the shape and size of the generated hole were irregular and nonuniform. While the front surface was excavated in a nearly circular fashion, the rear surface appeared to be irregularly punctuated with some surface cracks. This is manifest from Fig. 1(d) that shows the transmission-mode optical micrograph of another sample processed at the same condition. X-ray diffraction (XRD) patterns before and after irradiation are given in Fig. 1(e). The as-received Permalloy foil was highly textured with (111) and (200) orientations. After irradiation, the diffraction peaks, especially (111), became stronger and sharper. This indicates that the crystalline quality of the foil improved by laser irradiation. The laser-induced improvement of crystallinity is a common phenomenon that has been observed in many different materials. Together with the improved crystalline quality, a small $\mathrm{FeO}$ peak $^{21}$ was also observed in the irradiated sample. It seems evident that the Permalloy sample is partially oxidized as a result of the laser irradiation. Changing the energy of each pulse or the number of pulses did not produce circular holes either. This leads to a conclusion that a pulsed laser with low repetition rate and high pulse energy is not suitable for drilling metal foils and that a laser of low pulse energy and high repetition rate would be required.

Micromachining was then conducted using a nanosecond UV laser with variable repetition rate. Throughout the work, the repetition rate of the laser was set to $25 \mathrm{kHz}$. Experiments have first been carried out as to how many pulses are required to drill a hole. Fig. 2 shows transmission optical microscopic images taken after the sample was exposed to different numbers of pulses. At fixed pulse energy, a specific number of pulses were required to puncture a hole in the sample. As the number of pulses increased, the perforated hole became larger. However, the size of the hole did not increase in proportion to the number of pulses. Above a particular number, it remained nearly unchanged. This means that increasing the number of pulses above a certain threshold value should be avoided, because it simply enlarges the heat-affected zone.

Fig. 3 shows SEM images of the samples processed at different pulse energies with the number of pulses fixed at 20 . When the pulse energy was $60 \mu \mathrm{J}$, a circular concave hole was generated on the surface but the foil was not perforated. As the energy increased to $70 \mu \mathrm{J}$, the
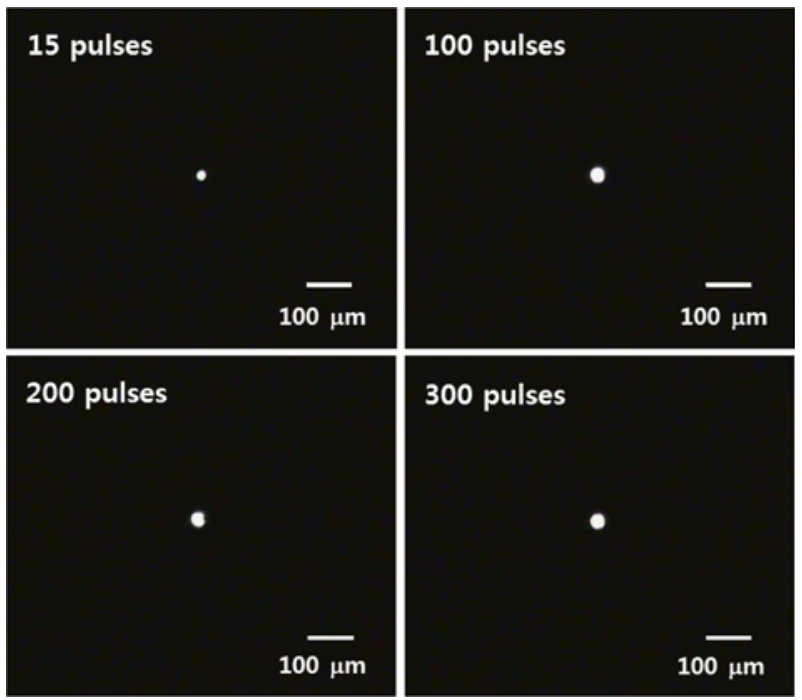

Fig. 2 Transmission optical micrographs taken after exposure to different numbers of UV laser pulses
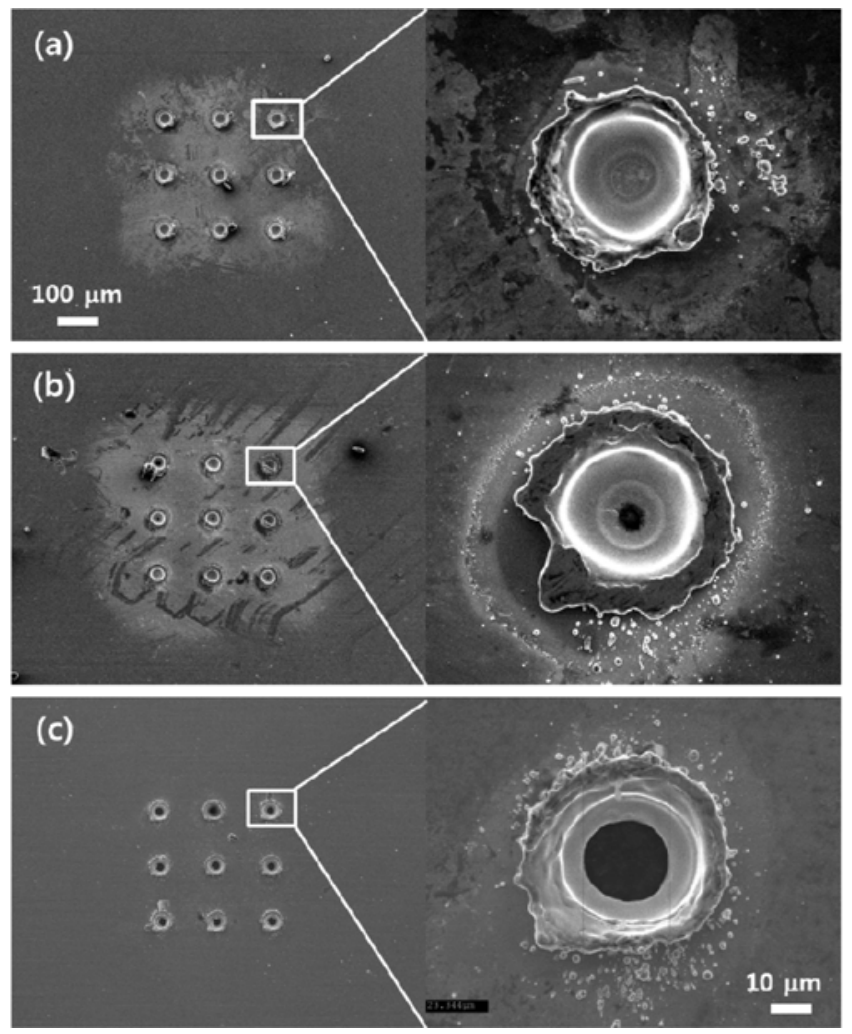

Fig. 3 SEM images of the samples processed with 20 pulses at different pulse energies of (a) $60 \mu \mathrm{J}$, (b) $70 \mu \mathrm{J}$, and (c) $90 \mu \mathrm{J}$

central part of this concave hole began to be punctured. The drilled hole became larger when the energy was further increased to $90 \mu \mathrm{J}$. At this condition, the size of the generated hole was about $23 \mathrm{~mm}$, which is smaller than the estimated laser spot size of $\sim 37 \mu \mathrm{m}$. Since the output beam has a Gaussian profile with $1 / \mathrm{e}^{2}$-size of $2.54 \mathrm{~mm}$, the edge of the beam will have significantly lower intensity than its center. Thus, the edge regions might have not contributed to the drilling. Fig. 4 plots the minimum pulse energy and number of pulses required to drill a hole in the Permalloy foil. The presented values are those needed to make a small initial aperture, not for drilling uniform holes. As expected, the 


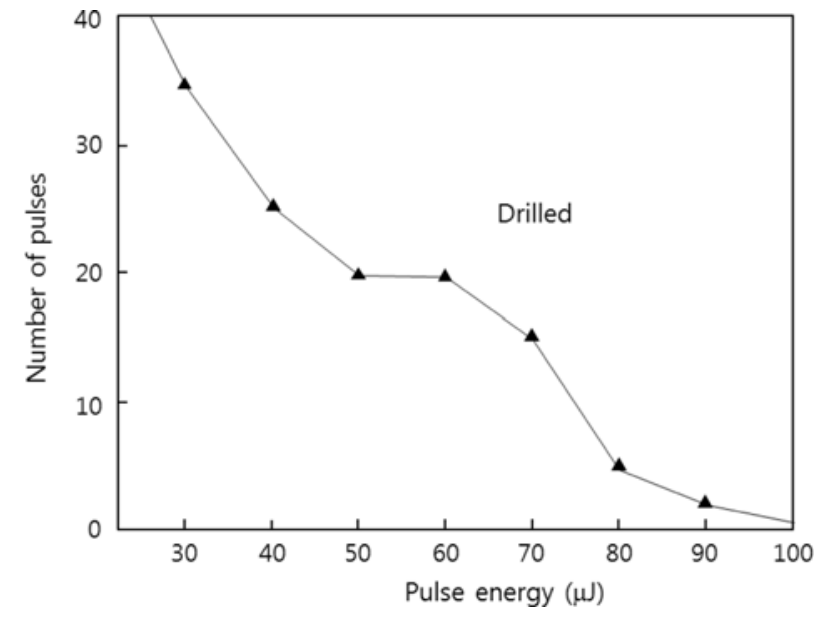

Fig. 4 Minimum pulse energy and number of pulses

required number of pulses decreased with increasing pulse energy. When the pulse energy was fixed, the uniformity of hole size on the front and rear surfaces improved as the number of pulses increased.

Laser drilling generally occurs through melting and vaporization (also referred to as "ablation") of the material that absorbs energy from a focused laser beam. ${ }^{22-24}$ The energy required to remove material by melting is less than that needed to vaporize the same volume. Therefore, a process that removes material by melting is often favored.

Whether melting or vaporization is more dominant depends on many factors, including laser pulse duration and energy. In most laser machining processes, ablation and melt expulsion coexist. Unless the drilling occurs by pure vaporization, some debris and burr are inevitable. As shown in Fig. 5(a), some melt/condensed particles along with drilling burr were observed around the generated hole. In an attempt to remove them, the processed foils were dipped in an $\mathrm{HCl} /$ $\mathrm{HNO}_{3}$ solution $\left(\mathrm{HCl} / \mathrm{HNO}_{3}=3 / 1\right.$ in volume). Since the scattered particles and burr were etched much faster than the matrix, we were able to obtain clear holes after etching for $30 \mathrm{~s}$ (Fig. 5(d)). As the inner wall of the drilled hole was also etched, the hole size somewhat increased to $\sim 30 \mu \mathrm{m}$ following the chemical process. Fig. 6 shows a fabricated hole pattern, in which each hole was exposed to 20 pulses at $90 \mu \mathrm{J}$, followed by etching for $30 \mathrm{~s}$. By careful adjustment of the sample height with respect to the focal position, we were able to reduce the hole size down to about $25 \mu \mathrm{m}$. The laser processing time required to drill 25 holes was $0.125 \mathrm{~s}$.

The results of XRD analysis are given in Fig. 7. The as-drilled sample exhibited a small $\mathrm{FeO}$ peak. A very tiny peak was observed at the same position even after etching for $30 \mathrm{~s}$. This indicates that the etched foil still contains an oxide phase. In fact, oxygen signal was detected by EDS in the vicinity of the hole. According to the EDS analysis, the oxygen content just near the hole was 10-20 at.\%. However, the signal intensity rapidly decayed with distance from the hole. No detectable signal was achieved at a distance of $70 \mu \mathrm{m}$ from the edge of the hole. While a nitrogen gas stream was blown to the irradiated region, it did not completely prevent the oxidation. It is not clear yet whether the oxidation is caused by thermal diffusion from the heated central area or direct exposure to the laser beam (note that the laser spot is bigger than the generated hole). To find the origin of oxidation and possibly avoid it, experiments using a more tightly
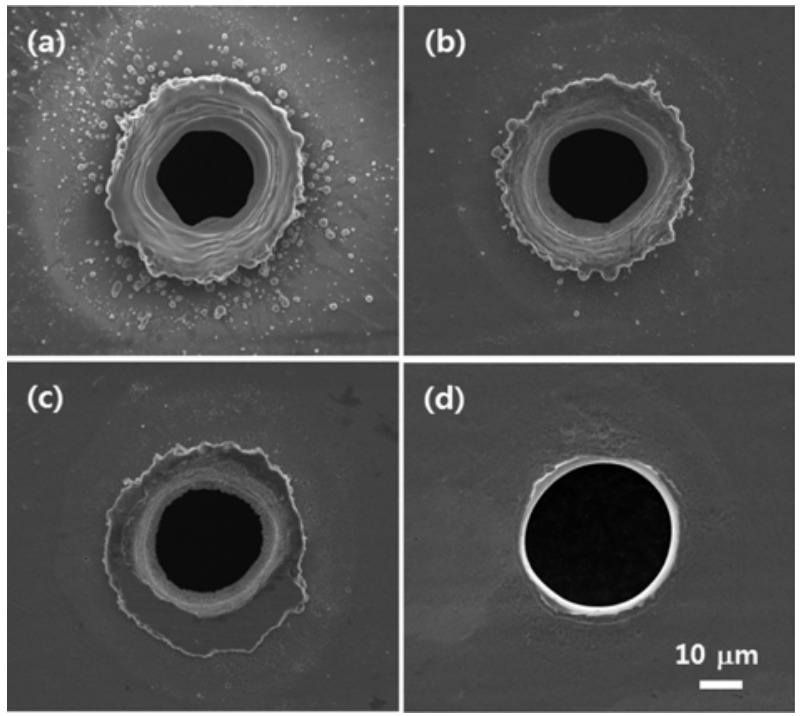

Fig. 5 Morphology change of the drilled hole with $\mathrm{HCl} / \mathrm{HNO}_{3}$ etching. (a) as-drilled, (b) after $10 \mathrm{~s}$ etching, (c) after $20 \mathrm{~s}$ etching, and (d) after $30 \mathrm{~s}$ etching. Before etching, the samples were drilled with 20 pulses at $90 \mu \mathrm{J}$

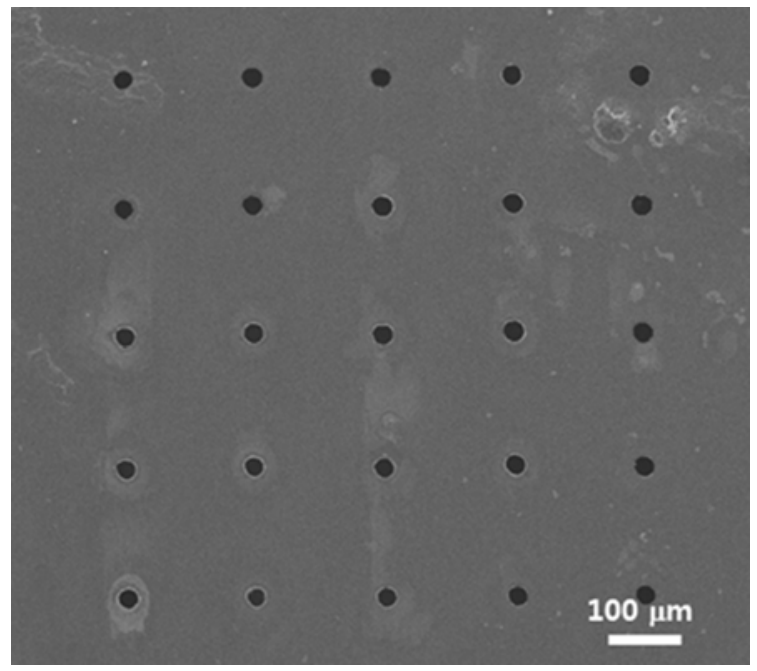

Fig. 6 Fabricated hole pattern

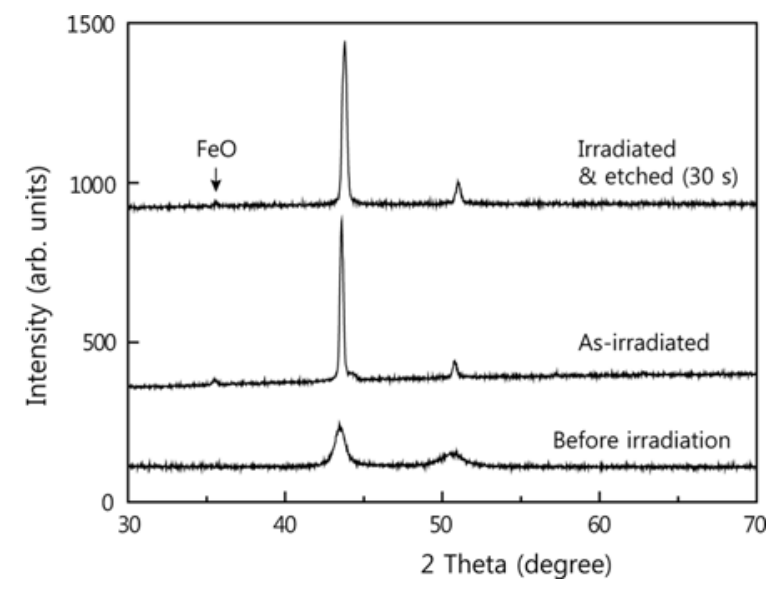

Fig. 7 XRD results

focused beam and/or a laser with ultrashort pulses would be necessary. This remains as an important topic of the future work. The 
manufacturing of many electronic devices relies on lithography-based subtractive processes. Direct deposition utilizing a fine metal mask would significantly reduce the cost and time required for device fabrication. In the current work, we showed that a clear hole structure with $25 \mu \mathrm{m}$ size could be obtained in the $12 \mu \mathrm{m}$-thick Permalloy foil. The fine metal mask for a display has rectangular holes, not circular, with specific requests to the corner radius. Therefore, this laser process should be further elaborated to be directly used for the targeted application. Nevertheless, the current article provides very encouraging results as a first step. It implies that the laser micromachining combined with a galvanometric scanner can be a viable tool for making fine metal masks required for the manufacturing of OLED and other devices.

\section{Conclusions}

In conclusion, we have investigated laser micromachining of Permalloy foils using two different types of nanosecond pulsed lasers. When an Nd:YAG laser with high pulse energy and low repetition rate was used, the sample was torned and cracked instead of being uniformly drilled. This is attributed to laser-induced thermal shock. It seemed that a high-energy beam focused on the surface caused abrupt heating and thermal expansion. On the contrary, crack-free holes could be fabricated by using a nanosecond UV laser with low pulse energy and high repetition rate. The number of pulses required for drilling decreased with increasing pulse energy. The size of the generated hole was smaller than the laser spot size, implying that only the central part of a focused Gaussian beam contributed to the drilling. Debris and burr incurring as a result of the laser micromachining could be quickly removed by chemical etching. This made it possible to obtain a clear drilled structure with $25 \mu \mathrm{m}$ hole size in the $12 \mu \mathrm{m}$-thick Permalloy foil. The presented laser-direct process may be a viable tool for fabricating fine metal masks for OLED and other devices.

\section{ACKNOWLEDGEMENT}

This work was supported by the Small \& Medium Business Administration of the Korea government (Contract number: C0146295).

\section{REFERENCES}

1. Lih, J. J., Chao, C. I., and Lee, C. C., "Novel Pixel Design for HighResolution AMOLED Displays with a Shadow Mask," Journal of the Society for Information Display, Vol. 15, No. 1, pp. 3-7, 2007.

2. Kim, S., Lee, S., Kim, M., Song, J., Hwang, E., et al., “A 3.0-in. 308-ppi WVGA AMOLED with a Top-Emission White OLED and Color Filter," Journal of the Society for Information Display, Vol. 17, No. 2, pp. 145-149, 2009.

3. Vaart, N., Lifka, H., Budzelaar, F., Rubingh, J., Hoppenbrouwers, J., et al., "Towards Large-Area Full-Color Active-Matrix Printed Polymer OLED Television," Journal of the Society for Information Display, Vol. 13, No. 1, pp. 9-16, 2005.
4. Shin, H., Kim, H., Lee, H., Yoo, H., Kim, J., et al., "Photoresist-Free Lithographic Patterning of Solution-Processed Nanostructured Metal Thin Films," Advanced Materials, Vol. 20, No. 18, pp. 3457-3461, 2008.

5. Kim, J., Kim, J., and Lee, M., "Laser Welding of Nanoparticulate $\mathrm{TiO}_{2}$ and Transparent Conducting Oxide Electrodes for Highly Efficient Dye-Sensitized Solar Cell," Nanotechnology, Vol. 21, No. 34, Paper No. 345203, 2010.

6. Joo, M., Lee, B., Jeong, S., and Lee, M., "Laser Sintering of $\mathrm{Cu}$ Paste Film Printed on Polyimide Substrate," Applied Surface Science, Vol. 258, No. 1, pp. 521-524, 2011.

7. Joo, M., Lee, B., Jeong, S., and Lee, M., "Comparative Studies on Thermal and Laser Sintering for Highly Conductive $\mathrm{Cu}$ Films Printable on Plastic Substrate," Thin Solid Films, Vol. 520, No. 7, pp. 2878-2883, 2012.

8. Kim, H., Shin, H., Ha, J., Lee, M., and Lim, K.-S., "Optical Patterning of Silver Nanoparticle Langmuir-Blodgett Films," Journal of Applied Physics, Vol. 102, No. 8, pp. 083505-083508, 2007.

9. Shin, H., Lee, H., Sung, J., and Lee, M., "Parallel Laser Printing of Nanoparticulate Silver Thin Film Patterns for Electronics," Applied Physics Letters, Vol. 92, No. 23, pp. 231107-231109, 2008.

10. Kim, S., Lee, H., and Lee, M., "Multi-Layered Ag Film Pattern Printed by Spatially Modulated Pulsed Laser Beam," Applied Surface Science, Vol. 257, No. 18, pp. 8013-8016, 2011.

11. Ghosh, A. and Mallik, A., "Unconventional Machining Processes," in: Manufacturing Science, $2^{\text {nd }}$ Ed., East-West Press, pp. 396-403, 2010.

12. Roos, S. O., "Laser Drilling with Different Pulse Shapes," Journal of Applied Physics, Vol. 51, No. 9, pp. 5061-5063, 1980.

13. Yang, B. and Lee, M., "Fabrication of Honeycomb Texture on PolySi by Laser Interference and Chemical Etching," Applied Surface Science, Vol. 284, pp. 565-568, 2013.

14. Yang, B. and Lee, M., "Mask-Free Fabrication of Inverted-Pyramid Texture on Single-Crystalline Si Wafer," Optics \& Laser Technology, Vol. 63, pp. 120-124, 2014.

15. Morikawa, H., Niinobe, D., Nishimura, K., Matsuno, S., and Arimoto, S., "Processes for Over 18.5\% High-Efficiency MultiCrystalline Silicon Solar Cell," Current Applied Physics, Vol. 10, No. 2, pp. S210-S214, 2010.

16. Niinobe, D., Morikawa, H., Hiza, S., Sato, T., Matsuno, S., et al., "Large-Size Multi-Crystalline Silicon Solar Cells with Honeycomb Textured Surface and Point-Contacted Rear toward Industrial Production," Solar Energy Materials and Solar Cells, Vol. 95, No. 1, pp. 49-52, 2011.

17. Rublack, T., Schade, M., Muchow, M., Leipner, H. S., and Seifert, G., "Proof of Damage-Free Selective Removal of Thin Dielectric Coatings on Silicon Wafers by Irradiation with Femtosecond Laser Pulses," Journal of Applied Physics, Vol. 112, No. 2, Paper No. 023521, 2012. 
18. Döring, S., Ullsperger, T., Heisler, F., Richter, S., Tünnermann, A., et al., "Hole Formation Process in Ultrashort Pulse Laser Percussion Drilling," Physics Procedia, Vol. 41, pp. 431-440, 2013.

19. Liu, Y., Wang, C., Li, W., Zhang, L., Yang, X., et al., "Effect of Energy Density and Feeding Speed on Micro-Hole Drilling in C/SiC Composites by Picosecond Laser," Journal of Materials Processing Technology, Vol. 214, No. 12, pp. 3131-3140, 2014.

20. Romoli, L., Rashed, C., Lovicu, G., Dini, G., Tantussi, F., et al., "Ultrashort Pulsed Laser Drilling and Surface Structuring of Microholes in Stainless Steels," CIRP Annals - Manufacturing Technology, Vol. 63, No. 1, pp. 229-232, 2014.

21. Boudinar, N., Djekoun, A., Chebli, A., Otmani, A., Bouzabata, B., et al., "X-ray Diffraction and Mossbauer Spectrometry Investigations of Invar Nanoparticles Produced by Mechanical Alloying," International Journal Nanoelectronics and Materials, Vol. 3, pp. 143153, 2010.

22. Chan, C. and Mazumder, J., "One-Dimensional Steady State Model for Damage by Vaporization and Liquid Expulsion due to LaserMaterial Interaction,” Journal of Applied Physics, Vol. 62, No. 11, pp. 4579-4586, 1987.

23. Kar, A. and Mazumder, J., "Two-Dimensional Model for Material Damage due to Melting and Vaporization during Laser Irradiation," Journal of Applied Physics, Vol. 68, No. 8, pp. 3884-3891, 1990.

24. Ganesh, R., Faghri, A., and Hahn, Y., "A Generalized Thermal Modeling for Laser Drilling Process - I. Mathematical Modeling and Numerical Methodology," International Journal of Heat and Mass Transfer, Vol. 40, No. 14, pp. 3351-3360, 1997. 